\title{
Augmenting propulsion demands during split-belt walking increases locomotor adaptation of asymmetric step lengths
}

\author{
Carly J. Sombric and Gelsy Torres-Oviedo * (D)
}

\begin{abstract}
Background: Promising studies have shown that the gait symmetry of individuals with hemiparesis due to brain lesions, such as stroke, can improve through motor adaptation protocols forcing patients to use their affected limb more. However, little is known about how to facilitate this process. Here we asked if increasing propulsion demands during split-belt walking (i.e., legs moving at different speeds) leads to more motor adaptation and more symmetric gait in survivors of a stroke, as we previously observed in subjects without neurological disorders.

Methods: We investigated the effect of propulsion forces on locomotor adaptation during and after split-belt walking in the asymmetric motor system post-stroke. To test this, 12 subjects in the chronic phase post-stroke experienced a split-belt protocol in a flat and incline session so as to contrast the effects of two different propulsion demands. Step length asymmetry and propulsion forces were used to compare the motor behavior between the two sessions because these are clinically relevant measures that are altered by split-belt walking.
\end{abstract}

Results: The incline session resulted in more symmetric step lengths during late split-belt walking and larger aftereffects following split-belt walking. In both testing sessions, subjects who have had a stroke adapted to regain speed and slope-specific leg orientations similarly to young, intact adults. Importantly, leg orientations, which were set by kinetic demands, during baseline walking were predictive of those achieved during split-belt walking, which in turn predicted each individual's post-adaptation behavior. These results are relevant because they provide evidence that survivors of a stroke can generate the leg-specific forces to walk more symmetrically, but also because we provide insight into factors underlying the therapeutic effect of split-belt walking.

Conclusions: Individuals post-stroke at a chronic stage can adapt more during split-belt walking and have greater after-effects when propulsion demands are augmented by inclining the treadmill surface. Our results are promising since they suggest that increasing propulsion demands during paradigms that force patients to use their paretic side more could correct gait asymmetries post-stroke more effectively.

Keywords: Stroke, Motor learning, Hemiparesis

\footnotetext{
* Correspondence: gelsyto@pitt.edu

Department of Bioengineering, University of Pittsburgh, 4420 Bayard Street,

Suite 110, Pitt, Pittsburgh, PA, USA
} 


\section{Background}

Brain lesions, such as stroke, may result in asymmetric gait, limiting patients' mobility and decreasing their quality of life [31]. Moreover, gait asymmetry can lead to comorbidities further affecting post-stroke gait such as musculoskeletal injuries [32] and joint pain [59]. Promising studies have shown that motor adaptation protocols forcing individuals to use their affected limb more, as in "constrained use therapy" [36], could lead to motor improvements. For example, split-belt walking (i.e., legs moving at different speeds), has been shown to reduce gait asymmetries post-stroke [7, 39-41, 47, 62, 63]. While this is encouraging, little is understood about the mechanisms underlying this process and how to facilitate it. This is relevant since not all individuals improve their gait following repeated exposure to split-belt walking $[7,61]$. Therefore, there is a scientific and clinical interest to identify factors that underlie the therapeutic effect of split-belt walking in order to augment rehabilitative effects.

Our previous work indicates that locomotor adaptation in young, unimpaired subjects increases by augmenting propulsion demands during split-belt walking. More specifically, greater propulsion demands during incline split-belt walking resulted in greater changes in step lengths relative to flat split-belt walking [70]. Individuals post-stroke have well-known deficits in paretic propulsion $[4,9]$ leading to asymmetric step lengths [65]. This deficient force generation raise the question of whether survivors of a stroke could increase their paretic propulsion to walk in the incline split-belt condition, and in turn exhibit greater adaptation of step length asymmetry during and after split-belt walking, as we observed in young, intact individuals [70]. We consider that this would be a possibility since there is evidence that survivors of a stroke can augment their propulsion forces when required by the walking condition; for example, when walking at fast speeds [3, 24-26, 34]. Thus, we tested whether the adaptation of step length asymmetry in survivors of a stroke could be augmented by increasing propulsion demands with incline split-belt walking.

We hypothesized that increasing propulsion demands during the split-belt condition by inclining the walking surface, which naturally augments propulsion forces [42, 43], would lead to greater adaptation of step length asymmetry during split-belt walking and larger aftereffects in individuals post-stroke. This was formulated on the basis of our results in young, unimpaired individuals [70]. To test our hypothesis, we performed a research study with subjects in the chronic phase poststroke, who experienced two split-belt adaptation protocols with distinct propulsion demands: a flat configuration and an incline configuration. We expected more step symmetry adaptation and greater after-effects following incline split-belt walking relative to flat splitbelt walking. We also anticipated that steady-state splitbelt walking and the following after-effects from each individual could be predicted from the subject-specific baseline gait; more specifically, from their baseline feet positions relative to the body at foot landing (i.e., leg orientations) needed to walk at the speed and inclination set on each leg in the split condition [70]. Therefore, we expected that baseline walking would be predictive of the step lengths achieved during split-belt walking and after-effects in an individual basis. These anticipated findings would suggest that therapies increasing bilateral propulsion demands during walking would be a good strategy for improving post-stroke gait.

\section{Methods}

We investigated the effect of augmenting propulsion demands during split-belt walking on gait adaptation under distinct slopes (i.e., flat and incline), which modulates propulsion forces $[42,43]$. To this end, we evaluated the adaptation and after-effects of 12 patients who have had a stroke (4 females, $60.3 \pm 10.0$ years of age) in the chronic phase of recovery ( $>6$ months post-stroke) during separate flat and incline testing sessions. Those who have had a stroke were eligible if they (1) had only unilateral and supratentorial lesions (i.e., without brainstem or cerebellar lesion) as confirmed by MRI, (2) were able to walk without assistance from others or a device for 5 min at a self-selected pace, (3) were free of orthopedic injury or pain that would interfere with testing, (4) had no other neurological condition other than stroke, (5) had no severe cognitive impairments defined by a MiniMental State Exam score below 24 [55], and (6) did not take medications that altered cognitive function. Overall, participants that met the inclusion criteria were mildly to moderately impaired post-stroke [13], as indicated by their Lower Extremity Fugl-Meyer score and walking speed (Table 1). Participants gave written and informed consent prior to participation. The University of Pittsburgh Institutional Review Board approved the experimental protocol experienced by all participants.

\section{General paradigm}

All subjects experienced a split-belt protocol while either walking flat or incline throughout two separate experimental sessions (Fig. 1a). The flat session was always performed first. The protocol was tailored (i.e., slope, duration, and speed) to each individual's ability so that each subject could complete both testing sessions at the same walking speed, given that walking speed directly affects propulsion forces. The subject-specific walking speed on the treadmill (i.e., the mid speed, which is reported in Table 1) was determined by subtracting 0.35 
Table 1 Clinical characteristics of stroke survivors

\begin{tabular}{|c|c|c|c|c|c|c|c|c|c|}
\hline $\mathrm{ID}$ & Age & Gender & $\begin{array}{l}\text { Affected } \\
\text { Side }\end{array}$ & Lesion Location & $\begin{array}{l}\text { Fugl-Meyer } \\
\text { Score }\end{array}$ & $\begin{array}{l}\text { Slow, Mid, Fast } \\
\text { Speed }(\mathrm{m} / \mathrm{s})\end{array}$ & $\begin{array}{l}\text { Total \# Adapt } \\
\text { Strides } \\
\text { (flat/ incline) }\end{array}$ & $\begin{array}{l}\text { Total \# } \\
\text { Post } \\
\text { Strides } \\
\text { (flat/ } \\
\text { incline) }\end{array}$ & $\begin{array}{l}\text { Incline } \\
\text { Session } \\
\text { Slope } \\
\left({ }^{\circ}\right)\end{array}$ \\
\hline P1 & 43 & Female & R & Left MCA and basal ganglia & 33 & $\begin{array}{l}0.75 \\
1.13 \\
1.50\end{array}$ & $907 / 609$ & $605 / 303$ & $8.5^{\circ}$ \\
\hline P2 & 55 & Female & $\mathrm{R}$ & $\begin{array}{l}\text { Left MCA and ACA, temporal lobe, } \\
\text { basal ganglia }\end{array}$ & 26 & $\begin{array}{l}0.54 \\
0.81 \\
1.08\end{array}$ & $867 / 301$ & $642 / 300$ & $5^{\circ}$ \\
\hline P3 & 64 & Female & $\mathrm{R}$ & $\begin{array}{l}\text { Left MCA, frontal, parietal lobe and } \\
\text { basal ganglia }\end{array}$ & 29 & $\begin{array}{l}0.40 \\
0.60 \\
0.80\end{array}$ & $617 / 368$ & $307 / 10$ & $5^{\circ}$ \\
\hline P4 & 58 & Female & R & Left medial, frontal and parietal area's & 21 & $\begin{array}{l}0.30 \\
0.45 \\
0.60\end{array}$ & $901 / 406$ & $625 / 10$ & $5^{\circ}$ \\
\hline P5 & 66 & Male & $\mathrm{R}$ & $\begin{array}{l}\text { Left MCA, frontal, temporal and } \\
\text { parietal lobes }\end{array}$ & 30 & $\begin{array}{l}0.51 \\
0.77 \\
1.02\end{array}$ & $606 / 452$ & $599 / 302$ & $5^{\circ}$ \\
\hline P6 & 60 & Female & $\mathrm{R}$ & Left frontal & 26 & $\begin{array}{l}0.60 \\
0.90 \\
1.20\end{array}$ & $907 / 597$ & $600 / 300$ & $5^{\circ}$ \\
\hline P7 & 77 & Male & $\mathrm{R}$ & Thalamus & 30 & $\begin{array}{l}0.23 \\
0.35 \\
0.47\end{array}$ & $589 / 605$ & $598 / 302$ & $5^{\circ}$ \\
\hline P8 & 59 & Male & $\mathrm{R}$ & Left MCA & 32 & $\begin{array}{l}0.47 \\
0.70 \\
0.93\end{array}$ & $905 / 608$ & $600 / 306$ & $8.5^{\circ}$ \\
\hline P9 & 52 & Male & $\mathrm{R}$ & Left MCA & 32 & $\begin{array}{l}0.64 \\
0.96 \\
1.28\end{array}$ & $903 / 602$ & $603 / 302$ & $5^{\circ}$ \\
\hline P10 & 66 & Male & L & $\begin{array}{l}\text { Right frontal superior, parietal and } \\
\text { posterior area's }\end{array}$ & 29 & $\begin{array}{l}0.51 \\
0.76 \\
1.01\end{array}$ & $908 / 519$ & $602 / 299$ & $8.5^{\circ}$ \\
\hline P11 & 75 & Male & $\mathrm{R}$ & $\begin{array}{l}\text { Left periventricular, temporal and } \\
\text { basal ganglia }\end{array}$ & 32 & $\begin{array}{l}0.63 \\
0.94 \\
1.25\end{array}$ & $913 / 497$ & $552 / 306$ & $5^{\circ}$ \\
\hline P12 & 49 & Male & $\mathrm{R}$ & Frontotemporal parietal & 33 & $\begin{array}{l}0.47 \\
0.71 \\
0.95\end{array}$ & $931 / 450$ & $303 / 300$ & $5^{\circ}$ \\
\hline
\end{tabular}

$\mathrm{m} / \mathrm{s}$ from each subject's overground walking speed during a Six-Minute Walking Test [64]. We selected this procedure to ensure all individuals completed the entire split-belt walking protocol [29]. The speeds experienced during split-belt walking were selected based on subject's mid walking speed. The slow speed was defined as $66.6 \%$ of the mid speed and the fast speed as $133.3 \%$ of the mid speed. These percentages were selected for two reasons: 1) to have a 2:1 split-belt ratio and 2) to have the same averaged speed (i.e., (slow + fast) $/ 2=$ mid speed) throughout all experimental epochs (i.e., baseline, adaptation, and post-adaptation). We selected an inclination of either $5^{\circ}$ or $8.5^{\circ}$ based on the level of physical fitness of each patient, which was qualitatively assessed with subjects' self-reported fatigue during the flat session. More specifically, participants reporting fatigue at the end of the flat session were set to walk with a $5^{\circ}$ slope during the inclined session, whereas those reporting continued energy at the end of the flat session were set to walk with an $8.5^{\circ}$ slope. This was done to ensure that all participants could complete the incline session. Both $5^{\circ}$ (e.g., $\left.[28,37,54,76]\right)$ and $8.5^{\circ}$ (e.g., $[42,70]$ ) have been utilized in previous work on the effect of sloped walking on human gait. The flat and incline sessions were separated by 204-360 days without split-belt walking to minimize the effect of multiple exposures to the splitbelt environment [45].

Experimental protocols for both sessions consisted of three epochs (i.e., Baseline, Adaptation, and PostAdaptation). These epochs were used to assess subjects' baseline walking characteristics and subjects' ability to adjust and recalibrate their gait for each session-specific 


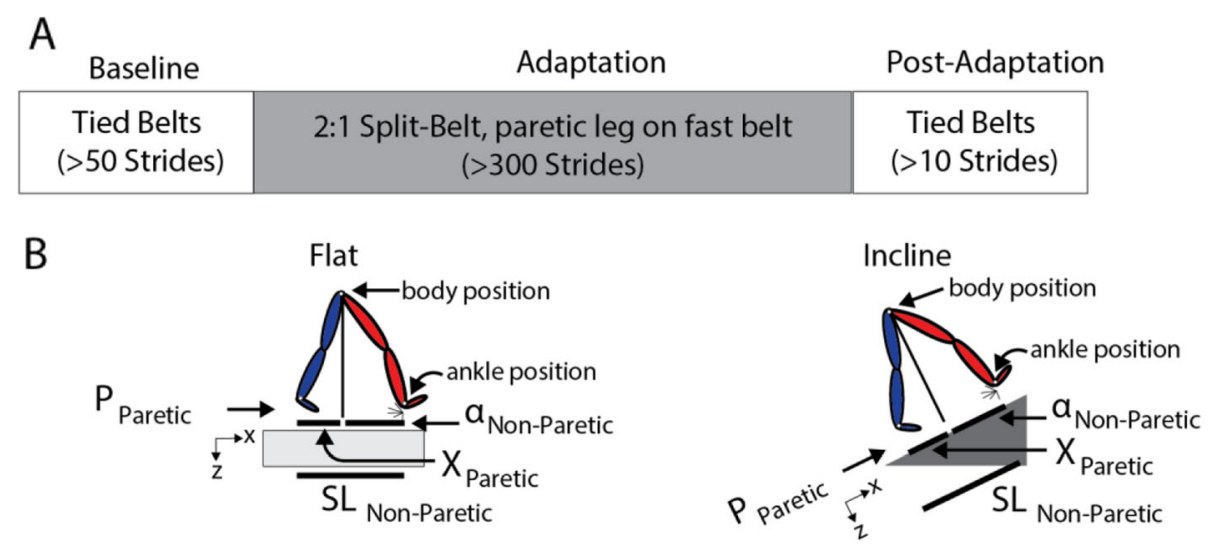

Fig. 1 Experimental Paradigm and Kinetic and Kinematic Analysis. a Paradigm used for both the flat and incline sessions to assess locomotor adaptation during and after split-belt walking. Subjects walked flat for the entire flat session, and incline (either $5^{\circ}$ or $8.5^{\circ}$ ) for the entire incline session. The walking speeds, duration of epochs, resting breaks and inclination were based on each subject's ability. $\mathbf{b}$ The decomposition of step length into leading $(a)$ and trailing $(X)$ leg positions with respect to the body is illustrated for each sloped condition. The body position, which is computed as the average of the greater trochanters, is illustrate with a white dot, and the perpendicular projection of the body onto the surface of the treadmill is illustrated as the black line coming from intersection of the legs. The position of the ankles is illustrated with white dots at the intersection of the foot and shank. This decomposition was done because it is known that inclination affects these aspects of step length differently $[15,16,46]$. Also note that when taking a step, the step length will depend on the position of the leading and trailing leg, which are generating a braking and propulsion force, respectively

slope. Subjects first experienced a baseline epoch, lasting at least 50 strides, to characterize their baseline gait at the specific slope used throughout each session. Subjects walked with both belts moving at the same mid speed (Table 1) in both the flat and incline sessions. A baseline epoch with the belts moving at the slow walking speed (i.e., $66.6 \%$ of the mid speed) was also measured during the flat session. However, the slow baseline epoch was removed in the incline session to ensure that all subjects could complete the entire protocol. Next, the Adaptation epoch of at least 300 strides (Table 1) was used to assess subjects' ability to adjust their gait in response to a splitbelt perturbation. During this epoch, the non-paretic leg walked twice as fast as the paretic leg. The paretic leg was defined as contralateral to the lesion site (which was visualized with MRI). The paretic leg always walked on the slow belt in the split-belt condition, even if this experimental design does not always reduce step length asymmetry post-stroke [12, 40, 41, 61, 62]. We chose this experimental design, rather than placing the paretic leg on the fast belt, because inclination augmented the slow leg's propulsion in young, intact subjects following the split-belt condition [70]. Thus, we focused our study on testing if incline split-belt walking would also augment the paretic propulsion in survivors of a stroke, who have known deficits generating paretic propulsion forces $[4,9]$. The duration of the adaptation epoch for each individual is presented in Table 1 . We had to tailor the duration of the adaptation epoch based on subjectspecific abilities. More specifically, we stopped the adaptation epoch if participants expressed or showed signs of fatigue. That is, we ended the adaptation epoch if participants either had difficulty maintaining their body position in the central region of the treadmill, if their heart rate reached $80 \%$ of their maximum heart rate (Max Heart Rate $=220$-subject's age $[22,23])$ for 50 consecutive strides, or if participants indicated the desired to stop walking. Despite this variation, all subjects exhibited remarkably similar results. Finally, The Post-Adaptation epoch, lasting at least 10 strides, was used to assess the after-effects when the split-belt condition was removed. Both belts moved at the same mid speed as in the Baseline epoch. We counted the number of strides in realtime to regulate the duration for each epoch, where a stride was defined as the period between two consecutive heel-strikes (i.e., foot landings) of the same leg. All participants took resting breaks as requested, except for the transition from split-to-tied walking where the belts were stopped and restarted as quickly as possible. No steps were taken during the resting breaks; subjects stood or sat still. Also, all subjects wore a safety harness to prevent falls. In addition, there was an instrumented handrail in front of the treadmill for balance support. The handrail data was used to quantify potential differences in handrail holding between the incline and flat sessions. Interestingly, the average force magnitude applied at the handrail was not different between the incline and flat sessions for Baseline $(p=0.75)$, Late Adaptation $(p=0.66)$, or After-Effects $(p=0.97)$. Also, 4 out of 12 individuals that held on to the handrail throughout each experimental session did not qualitatively exhibit smaller changes in adaptation or after- 
effects across sessions than their counterparts and were, therefore, included in the study.

\section{Data collection}

Kinematic and kinetic data were used to characterize subjects' ability to adapt their gait during Adaptation, and retain the learned motor pattern during PostAdaptation. Kinematic data were collected with a passive motion analysis system at $100 \mathrm{~Hz}$ (Vicon Motion Systems, Oxford, UK). Subjects' behavior was characterized with passive reflective markers placed symmetrically on the ankles (i.e., lateral malleolus) and the hips (i.e., greater trochanter) and asymmetrically on the shanks and thighs (to differentiate the legs). The origin of the kinematic data was rotated with the treadmill in the incline conditions such that the z-axis ('vertical' in the flat condition) was always orthogonal to the surface of the treadmill (Fig. 1b). Gaps in raw kinematic data were filled with a quintic spline interpolation (Woltring; Vicon Nexus Software, Oxford Uk). Kinetic data were collected with an instrumented split-belt treadmill at $1000 \mathrm{~Hz}$ (Bertec, Columbus, OH). Force plates were zeroed prior to each testing session so that each force plate's weight did not affect the kinetic measurements. In addition, the reference frame was rotated at the session-specific inclination such that the anteriorposterior forces were aligned with the surface on which the subjects walked. A heel-strike was identified in realtime when the raw normal force under each foot reached a threshold of $30 \mathrm{~N}$. This threshold was chosen to ensure accurate counting of strides at all slopes. During data processing we used a threshold of $10 \mathrm{~N}$ on median filtered data (with a $5 \mathrm{~ms}$ window) to detect the timing of heel strikes more precisely.

\section{Data analysis}

\section{Kinematic data analysis}

Kinematic behavior was characterized with step length asymmetry, which exhibits robust adaptation in split-belt paradigms (e.g., [60]) and is of clinical interest [31, 32, 59]. It is calculated as the difference in step length between the two legs on consecutive steps (EQ.1). Step length (SL) is defined as the anterior-posterior (i.e., along the $x$-axis) distance in millimeters between the ankle markers at forward leg heel strike (e.g., paretic step length is defined as the distance between the two ankle markers at the paretic heel strike). Therefore, equal step lengths result in zero step length asymmetry. A positive step length asymmetry indicates that the non-paretic leg's step length was longer than the paretic leg's step length. Step length asymmetry was normalized by stride length, which is the sum of two consecutive step lengths, resulting in a unitless parameter that is robust to intersubject differences in step size. This is particularly relevant when averaging step length asymmetries across subjects since they were walking at different speeds.

$$
\begin{aligned}
\text { step length asymmetry } & =\frac{S L_{\text {Non-Paretic }}-S L_{\text {Paretic }}}{S L_{\text {Non-Paretic }}+S L_{\text {Paretic }}} \\
& =\frac{S L_{\text {Fast }}-S L_{\text {Slow }}}{S L_{\text {Fast }}+S L_{\text {Slow }}}
\end{aligned}
$$

Each step length was also decomposed into anterior and posterior foot distances relative to the hip position (the average of the greater trochanter positions; Fig. 1b) as in previous work [20]. This was done to quantify the leading and trailing legs' positions relative to the body when taking a step because inclination is known to affect these measures $[16,46]$. The leading leg's position (' $\alpha$ ') was computed as the anterior-posterior (i.e., along the $x$ axis) distance in millimeters between the leading leg's ankle and the hip at heel strike; similarly, the trailing leg's position (' $\mathrm{X}$ ') was computed as the anteriorposterior (i.e., along the $\mathrm{x}$-axis) distance in millimeters between the trailing leg's ankle and the hip at heel strike. The hip position, which is a proxy for the body's position, was estimated as the mean instantaneous position across hip markers. By convention positive $\alpha$ values indicate that the foot landed in front of the hips, whereas negative $\mathrm{X}$ values indicate that the trailing leg was behind the hips. Note that the magnitudes of $\alpha$ and $X$ summed to the leading leg's step length. As indicated in Fig. $1 \mathrm{~b}, \alpha$ and $\mathrm{X}$ were computed aligned to the treadmill's surface in all sloped conditions.

\section{Kinetic data analysis}

Kinetic data were used to characterize the adaptation of ground reaction forces (GRF). We focused our analysis on the propulsion component of the anterior-posterior GRF because they are associated with walking speed, hemiparetic severity [9], and step length asymmetry [4]. The anterior-posterior GRF (AP forces) were first lowpass filtered with a cutoff frequency of $20 \mathrm{~Hz}$. Then, forces in Newtons were normalized by each subject's body weight in kilograms to get a unitless measure that is robust to difference in subjects' body weight. Similar to previous studies reporting the effect of sloped walking on human gait $[28,42]$ and previous split-belt studies $[53,57,70]$ we computed peak propulsion forces as the maximum AP force $\left(\mathrm{P}_{\text {Paretic }}\right.$ and $\left.\mathrm{P}_{\text {Non-Paretic }}\right)$ excluding the initial positive AP forces following heel strike. Note that we did not remove slope-specific biases due to gravity because we focused on analyzing changes in propulsion forces between epochs of interest. The anterior-posterior kinetic data for one leg for a single subject during the flat testing session was lost due to a hardware malfunction. Thus, analysis of the paretic 
propulsion forces was performed with 11 subjects rather than 12 subjects.

\section{Kinetic and kinematic outcome measures}

We computed 5 outcome measures for each kinetic and kinematic parameter: Baseline, Late Adaptation, AfterEffects, $\Delta$ Adapt, and $\Delta$ Post. These 5 outcome measures were computed for the flat session and the incline session. In brief, these outcome measures were used to characterize regular walking (i.e., Baseline) and changes during the Adaptation (i.e., Late Adaptation) and PostAdaptation epoch (i.e., After-Effects) relative to the Baseline epoch. Finally, we also used these measures to characterize gait changes within either the Adaptation epoch (i.e., $\Delta$ Adapt) or the Post-Adaptation epoch (i.e., $\Delta$ Post). More specifically, the outcome measure called Baseline was quantified as the average of the last 40 strides of the mid speed Baseline epoch, as in previous studies $[27,29,70]$. This was done to characterize each participants' gait prior to adaptation in every session. We used these Baseline measures for each session (i.e., the flat and for the incline session) to characterize changes in step length asymmetry and propulsion forces beyond those observed by walking incline (without the split condition). Thus, Late Adaptation was defined as the difference between the average of the last 40 strides of the Adaptation epoch relative to the baseline behavior in each session (i.e., outcome measure called Baseline). The outcome measure called Late Adaptation indicated the steady state behavior reached at the end of the Adaptation epoch and it was characterized with the last 40 strides for consistency with previous work [14, 21, 29, $40,44,48,49,56,62,72]$. The outcome measure labelled After-Effects was defined as the difference between the average of the first 5 strides of Post-Adaptation (i.e., Early Post-Adaptation) and the Baseline measure (i.e., early Post-Adaptation - Baseline), as in previous studies $[14,21,29,40,44,48,49,56,62,72]$. Positive AfterEffect values indicated increments in magnitude of a specific parameter during the Post-Adaptation epoch relative to the Baseline epoch, and vice versa for negative values. We also characterized the behavioral changes within Adaptation and Post-Adaptation with $\Delta$ Adapt and $\Delta$ Post, respectively. $\Delta$ Adapt was computed as the difference between Late Adaptation and Early Adaptation (i.e., average of the first 5 strides during the Adaptation epoch, as in previous studies [14, 19, 29, 40, 44, 48, $49,51,56,72]$. $\Delta$ Post was computed as the difference between Baseline and early Post-Adaptation (e.g., Baseline - Early Post-Adaptation). Baseline was used instead of late Post-Adaptation because the duration of the Post-Adaptation epoch was not sufficiently long in all individuals to extinguish split-belt After-Effects. Thus, Baseline behavior was used a proxy for the late Post-
Adaptation behavior. We considered this to be an adequate estimation of the overall changes that occurred in Post-Adaptation, but this is a limitation of our analysis. Therefore, readers should consider the possibility that $\Delta$ Post values might be smaller than those reported since people might not return to their baseline behavior after a long period of walking (not recorded). $\Delta$ Adapt and $\Delta$ Post were calculated such that an increase in the magnitude of a parameter resulted in positive values and vice versa.

\section{Statistical analysis}

A significance level of $\alpha=0.05$ was used for all statistical tests, which were performed either with Stata (StataCorp LP, College Station, TX) or with MATLAB (The MathWorks, Inc., Natick, Massachusetts, United States). Normality was assessed with the Lilliefors Test. Except for the step length asymmetries during baseline, all of our step length asymmetry and propulsion outcome measures were normally distributed, thus parametric testing was utilized.

\section{Group analyses}

We tested the effect of slope on step length asymmetry and propulsion forces. In all statistical analyses we used unbiased values. This was done to identify slope-related differences beyond changes in baseline gait features. We used paired t-tests to compare outcome measures (e.g., $\triangle$ Adapt, $\Delta$ Post, Late Adaptation, After-Effects) in the flat vs. incline sessions for all gait parameters. We quantified the effect size with a Cohen's difference, $d$, which is appropriate for paired data [17]. In addition, we tested if step length values post-adaptation (i.e., $\Delta$ Post) were significantly different from zero at each slope with onesample t-tests, where each $p$-value was corrected for multiple comparisons with Bonferroni corrections.

During baseline walking, it was also of interest to identify differences between the paretic and non-paretic propulsion forces in addition to determining the effect of slope on outcome measures. Therefore, we performed ANOVAs with individual subjects as a random factor to account for the paired nature of the data set and slope and leg as fixed, repeated factors. Effect sizes $\left(\eta^{2}\right)$ were computed for each factor. These ANOVAs were performed on the peak propulsion values and the trailing leg's position because our study was focused on the propulsion phase of the gait cycle, which is associated to these two parameters.

The changes of both step lengths during the Adaptation and Post-Adaptation epochs for the flat and incline sessions was also of interest. Therefore, we performed an ANOVA with individual subjects as a random factor to account for the paired nature of the data, and the fixed factors are slope, leg, and epoch. Slope and leg 
were considered repeated factors in the analysis. Epoch is not repeated and is treated as a between-subject factor given that these epochs are not directly associated [70]. Effect sizes $\left(\eta^{2}\right)$ were computed for each factor.

\section{Regression analyses}

We tested the association between leg positions (' $\alpha$ ' and ' $\mathrm{X}$ ') during speed-specific Baseline and Late Adaptation to determine if Late Adaptation values could be predicted from Baseline values in survivors of a stroke, as observed in young unimpaired subjects [70]. We specifically tested the model $|y|=a_{*}|z|$, where $y$ is the predicted leg position during Late Adaptation and $z$ is the leg position recorded during Baseline. We also tested the ipsilateral association between $\alpha$ s during Late Adaptation and Post-Adaptation and the contralateral association between Xs during these epochs in survivors of a stroke, since these relations were also observed in young, intact individuals [70]. Thus, we tested the model $|y|=a_{*}|z|$, where $y$ is each leg's position during Early Post-Adaptation and $\mathrm{z}$ is either the ipsilateral ' $\alpha$ ' position recorded during Late Adaptation or the contralateral ' $\mathrm{X}$ ' position recorded during Late Adaptation. An absolute value of $z$ was utilized so that the data would not bias the results of the regression to be linear by having a cluster of positive $(\alpha)$ and negative $(\mathrm{X})$ data points.
Pearson correlation coefficients $(r)$ are presented as an indicator of effect size.

\section{Results \\ Adaptation and recalibration of step length asymmetry are augmented when walking incline}

Step length asymmetry adaptation and recalibration were augmented by incline walking compared to flat walking. Figure 2a illustrates the evolution of step length asymmetry throughout the flat and incline sessions. Fig. $2 \mathrm{~b}$ indicates that there was a wide range of individual Baseline step length asymmetries (colored lines, positive values indicate that the non-paretic step length is longer than the paretic step length) and slope did not change the group average biases $(p=0.30, \mathrm{t}(11)=1.08$, $\mathrm{d}=0.15$ ). During Adaptation, participants exhibited similar changes in step length asymmetry from early to late Adaptation (Fig. 2d, $p=0.75, \mathrm{t}(11)=0.33, \mathrm{~d}=0.08$ ), but they were more symmetric in the incline than the flat session in Late Adaptation (Fig. 2c, $p=0.004$, $\mathrm{t}$ $(11)=-3.68, d=0.98)$. Furthermore, the incline session had larger magnitudes of After-Effects during early PostAdaptation relative to the flat session (Fig. 2e, $p=0.008$, $\mathrm{t}(11)=-3.21, \mathrm{~d}=1.08)$. Thus, incline walking augmented step length symmetry during Late Adaptation and the magnitude of After-Effects.

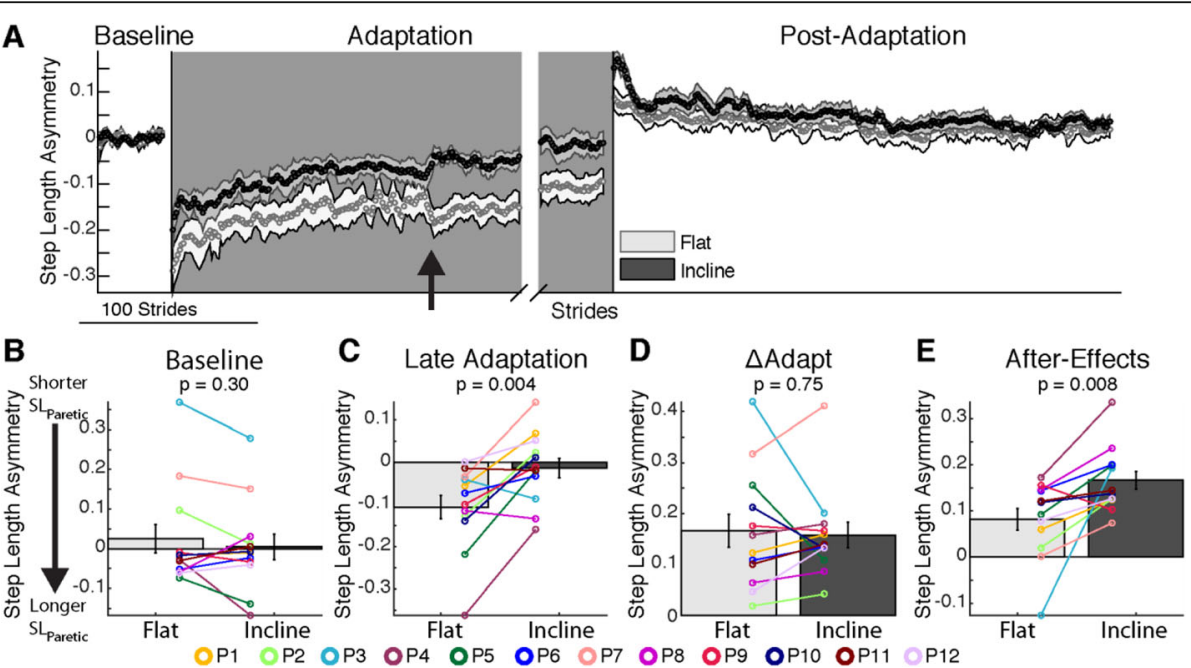

Fig. 2 Step Length Asymmetry Adaptation and Recalibration. a Stride-by-stride time course of step length asymmetry during Baseline, Adaptation, and Post-Adaptation for each session are shown. Note that each subject's baseline bias has been removed, resulting in average step length asymmetry values of zero during Baseline. Each data point represents the average of 5 consecutive strides and shaded regions indicate the standard error for each session. For display purposes only, we include in the time courses stride values that were computed with a minimum of 10 subjects and the late adaptation behavior is aligned to the end of each subject's adaptation epoch. The black arrow indicates a discontinuity in the data caused by many subjects taking a resting break at the same time. b-e The height of the bars indicates group average step length asymmetry \pm standard errors. Individual subjects are represented with colored dots connected with lines. $\mathbf{b}$ Baseline: Baseline step length asymmetry is not influenced by slope. c Late Adaptation: Note that each session plateaued at different step length asymmetry values during the Adaptation epoch such that subjects reached more symmetric step lengths in the incline session than the flat session (d) $\Delta$ Adapt: Participants changed their gait by similar amounts during the Adaptation epoch in both sessions. e After-effects: Subjects had larger After-Effects during early Post-Adaptation in the incline session than the flat session, which is consistent with the Late Adaptation differences across sessions 
Both step lengths contribute to step length asymmetry adaptation and after-effects during incline walking in the asymmetric motor system

Survivors of a stroke adjusted both step lengths during split-belt walking. The survivors of a stroke modulate both their slow (paretic) and fast (non-paretic) step lengths during Adaptation and have After-Effects during Post-Adaptation (Fig. 3a). The change of each step length during the Adaptation and Post-Adaptation epochs are quantified in Fig. 3b. There was a significant effect of epoch $\left(\mathrm{p}_{\text {epoch }}=0.001, \mathrm{~F}_{\text {epoch }}(1,22)=13.54, \eta^{2}=\right.$ 0.38 ) and interaction between leg and epoch $\left(\mathrm{p}_{\text {leg\#epoch }}<\right.$ $\left.0.001, F_{\text {leg\#epoch }}(1,22)=94.23, \eta^{2}=0.81\right)$ indicating that the step length with the paretic leg is reduced during Adaptation, but increased during Post-Adaptation and vice versa for the non-paretic leg. Overall, slope did not alter step length changes $\left(\mathrm{p}_{\text {slope }}=0.16, \mathrm{~F}_{\text {slope }}(1,22)=\right.$ $2.14, \eta^{2}=0.09, \mathrm{p}_{\text {leg }}=0.44, \mathrm{~F}_{\text {leg }}(1,22)=0.61, \eta^{2}=0.03$, $\mathrm{p}_{\text {slope\#leg }}=0.18, \mathrm{~F}_{\text {slope\#leg }}(1,22)=1.88, \eta^{2}=0.08$, pslope\#epoch $\left.=0.17, \mathrm{~F}_{\text {slope\#epoch }}(1,22)=1.97, \eta^{2}=0.08\right)$, except for the paretic leg's de-adaptation as quantified by $\Delta$ Post $\left(\mathrm{p}_{\text {leg\#epoch\#slope }}=0.016, \mathrm{~F}_{\text {leg\# } \text { epoch\#slope }}(1,22)=6.86, \eta^{2}=\right.$ $0.24)$. More specifically, the paretic step lengths did not exhibit de-adaptation in the flat session (i.e., $\Delta$ Post is not different from zero, porrected $=1.00, \mathrm{t}(11)=0.92)$, whereas step lengths for both legs had significant deadaptation in the incline session (i.e., non-zero $\Delta$ Post, $\mathrm{p}_{\text {Corrected }} \leq 0.005$, t $\left.(11) \geq|4.3|\right)$. Survivors of a stroke use both their paretic and non-paretic leg to counteract the split-belt perturbation and both legs are recalibrated following incline adaptation.

\section{Slope and speed-specific walking demands determine the distinct step length asymmetries across inclination conditions}

Speed and slope-specific leg orientations mediated the distinct step length asymmetries selected during Late Adaptation and early Post-Adaptation. Figure 4a illustrates a top-down view of the baseline leg orientations that contribute to each step length relative to the hips. While we found significantly different leg orientations across individuals (colored lines, $\mathrm{p}_{\text {Individual }}=0.002, \mathrm{~F}_{\text {Indivi- }}$ dual $\left.(11,11)=6.99, \eta^{2}=0.87\right)$, we observed that the trailing leg position, $\mathrm{X}$, was larger in the incline than flat condition for both legs $\left(\mathrm{p}_{\text {Slope }}=0.042, \mathrm{~F}_{\text {Slope }}(1,11)=\right.$ $5.32, \eta^{2}=0.33, \mathrm{p}_{\text {Leg }}=0.22, \mathrm{~F}_{\text {Leg }}(11,11)=1.72, \eta^{2}=0.14$, $\left.\mathrm{P}_{\text {Slope\#Leg }}=0.76, \mathrm{~F}_{\text {Slope\#Leg }}(1,11)=0.10, \eta^{2}=0.01\right)$. The schematic in Fig. 4b illustrates the relation between

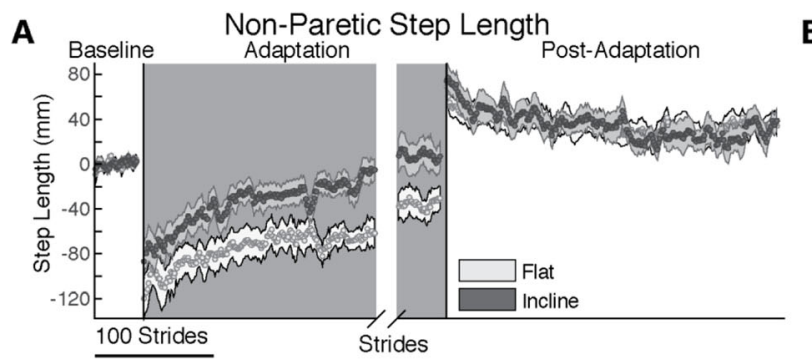

B
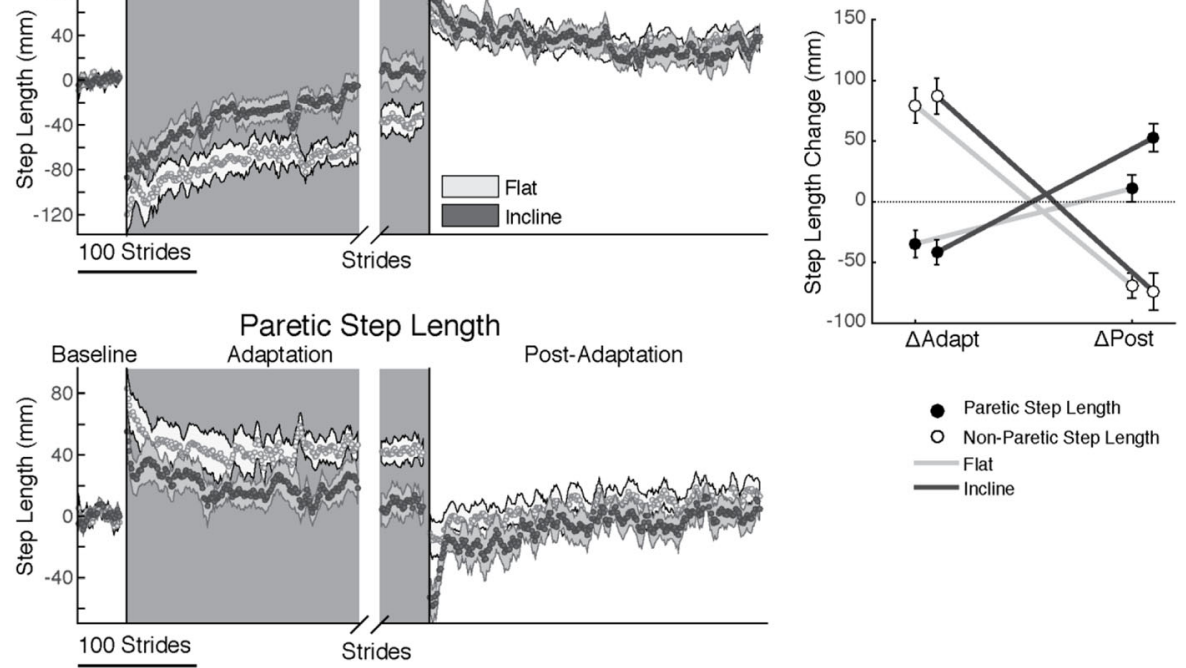

Fig. 3 Step length Adaptation and After-Effects. a Time courses of step lengths when stepping with either the non-paretic leg (top panel, fast leg during Adaptation) or the paretic leg (bottom panel, slow leg during Adaptation) during three epochs: Baseline, Adaptation, and Post-Adaptation. Note that each subject's baseline bias has been removed, resulting in average step length values of zero during Baseline. The negative values in the non-paretic step lengths indicate that on average subjects are taking shorter steps with the non-paretic leg relative to baseline walking, whereas the opposite is observed with the paretic one. Each data point represents the average of 5 consecutive steps and shaded regions indicate the standard error for each group. For display purposes only, we include averaged values during Post-Adaptation that were computed with a minimum of 10 subjects and the late adaptation behavior is aligned to the end of each subject's adaptation epoch. $\mathbf{b}$ The effect of slope on each leg's change during Adaptation ( $\Delta$ Adapt) and Post-Adaptation ( $\Delta$ Post) is illustrated. Note that both the paretic and non-paretic leg adapted similarly. While the non-paretic leg has recalibrated $(\Delta$ Post $\neq 0)$ following both the flat and incline session, the paretic leg is only recalibrated following incline Adaptation 
A
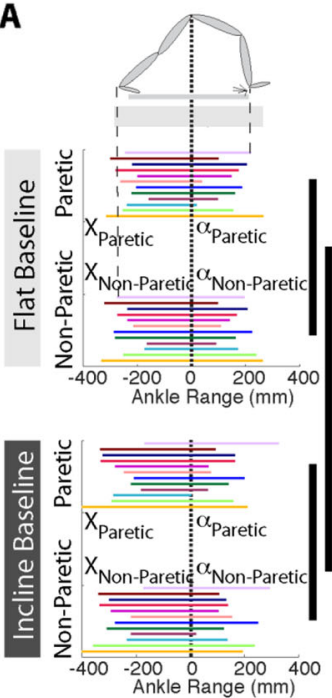

OP1 OP3 OP5 OP7 OP9 OP11

OP2 OP4 OP6 OP8 OP10 OP12
B Slow Baseline Fast Baseline D

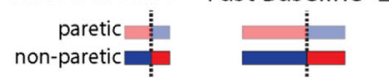

Late Adaptation

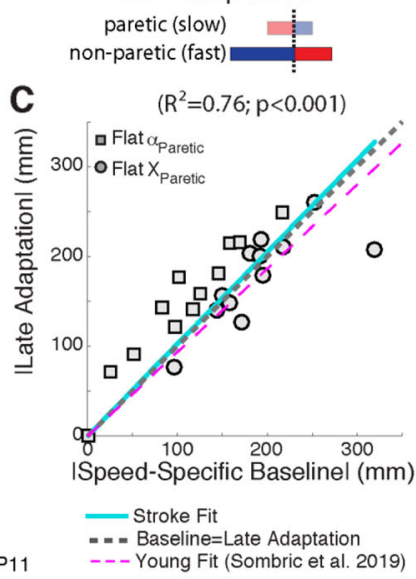

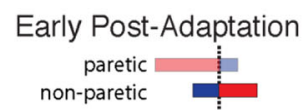

Fig. 4 Leg orientation Adaptation and After-Effects. a Leg orientations are depicted for individual subjects (as indicated with different colors) in both the flat and incline conditions. Note that subjects orient their legs about their bodies differently and that leg orientations are based on slope. Thick vertical black lines indicated a significant effect of leg (i.e., paretic or non-paretic) and slope (i.e., flat or incline) on trailing leg positions. $\mathbf{b}$ Schematic of the slow and fast (predicted) baseline behavior for the paretic and non-paretic leg orientations, respectively. The speedspecific leg orientations were regained during Late Adaptation. c The similarity between leg orientations across the speed-specific Baseline and Late Adaptation epochs is illustrated by the significant regression (solid cyan line; $|y|=a *|x|, 95 \%$ confidence interval for $a=[0.92,1.13]$ ). Recall that a slow Baseline was only collected in the flat session, thus only the slow Baseline and Late Adaptation for the paretic leg (which walked slow during Adaptation) are shown. Note that the regression line closely overlaps with the idealized situation in which baseline and late adaptation values are identical (dashed gray line; i.e., $y=x$ ) and the behavior of young, healthy adults ([70], dashed magenta line). $\mathbf{d}$ Schematic of the leg orientations during early Post-Adaptation. The forward leg positions are ipsilaterally and the trailing leg positions are contralaterally maintained from split-to-tied walking. e The ipsilateral and contralateral similarity between $a$ and $X$, respectively, across the Late Adaptation and early PostAdaptation epochs is quantified with a significant correlation (solid cyan line; $|y|=a *|x|, 95 \%$ confidence interval for $a=[0.94,1.02]$ ). The idealized situation in which Late Adaptation and early Post-Adaptation values are identical (dashed gray line; i.e., $y=x$ ) and the behavior of young, healthy adults ([70], dashed magenta line) are presented as a reference

baseline speed-specific leg orientations and late Adaptation [70]. Figure 4c indicates that the participants' leg orientations during slow Baseline walking predict well those achieved during late Adaptation (solid cyan line; $|\mathrm{y}|=\mathrm{a}^{*}|\mathrm{x}| ; 95 \%$ confidence interval of $\mathrm{a}=[0.92,1.13]$, $\left.\mathrm{t}_{\text {slope }}(95)=20.0, \mathrm{R}^{2}=0.76, p<0.001, \mathrm{r}=0.84\right)$. We also show as a reference, the relation between (recorded) Baseline and (predicted) Late Adaptation leg orientation values for both legs and both inclinations in young unimpaired individuals [70] (magenta dashed line; $(|y|=$ $\mathrm{a}^{*}|\mathrm{x}| ; 95 \%$ confidence interval of $\mathrm{a}=[0.91,0.96], \mathrm{t}_{\text {slope }}$ $\left.(95)=73.04, R^{2}=0.89, p<0.001, r=0.94\right)$. Note the similarity between the intact and lesioned behavior (cyan vs. magenta lines).

Moreover, we found that the leg orientations achieved during Late Adaptation were predictive of subjects' PostAdaptation behavior (Fig. 4d). Specifically, the leading leg's orientations were similar before and after removal of the split-belt perturbation (i.e., Late Adaptation $\alpha_{\text {Pare- }}$ tic $=$ Post-Adaptation $\alpha_{\text {Paretic }}$ and vice versa) whereas the trailing legs' orientations were swapped between the legs (i.e. Late Adaptation $\mathrm{X}_{\text {Paretic }}=$ Post-Adaptation $\mathrm{X}_{\text {Non-Pare- }}$ tic and vice versa). This is supported by the significant relationship between Late Adaptation and PostAdaptation leg orientations observed when individual subjects' values for each leg and both sloped sessions are regressed (Fig. 4e; solid cyan line; $|\mathrm{y}|=\mathrm{a}^{*}|\mathrm{x}| ; 95 \%$ confidence interval of $\mathrm{a}=[0.94,1.02], \mathrm{t}_{\text {slope }}(95)=47.5, \mathrm{R}^{2}=$ $0.79, p<0.001, r=0.86)$. We also show as a reference, the relation between (recorded) Late Adaptation and (predicted) Post-Adaptation leg orientation values for both legs and both sloped conditions in young, intact individuals (magenta dashed line; $\left(|\mathrm{y}|=\mathrm{a}^{*}|\mathrm{x}| ; \quad 95 \%\right.$ confidence interval of $\mathrm{a}=[0.95,1.03], \mathrm{t}(95)=47.54$, $\left.\mathrm{R}^{2}=0.78, p<0.001, \mathrm{r}=0.87\right)$. Note the similarity between the intact and lesioned behavior (cyan vs. magenta lines). Similar to the intact motor system, the lesioned motor system is able to recover speed and slope-specific leg orientations during Late Adaptation, which predict aftereffects during Post-Adaptation. 


\section{Larger after-effects of propulsion forces following split- belt incline walking}

Sloped walking influenced the extent of recalibration of the non-paretic propulsion forces. Figure 5a shows that propulsion forces were altered during the Adaptation epochs. These data are plotted relative to Baseline propulsion forces (i.e., mid speed), which were larger in the incline condition and the non-paretic leg for both sloped conditions (Fig. 5b: $\mathrm{P}_{\text {Individual }}=0.007, \mathrm{~F}_{\text {Individual }}(11,10)=$ $4.84, \eta^{2}=0.83$, plope $<0.0001, \mathrm{~F}_{\text {Slope }}(1,10)=4.84, \eta^{2}=$ $0.85, \mathrm{p}_{\text {Leg }}=0.040, \mathrm{~F}_{\text {Leg }}(1,10)=5.42, \eta^{2}=0.33$, p $\mathrm{p}_{\text {Slope\#- }}$ Leg $\left.=0.43, \mathrm{~F}_{\text {Slope\#Leg }}(1,10)=0.69, \eta^{2}=0.06\right)$. Note that subjects were closer to generating Baseline-like propulsion forces during Late Adaptation in the incline session compared to the flat session for both legs, resulting in larger Late Adaptation paretic propulsion forces in the incline session (Fig. 5c). Even though the Late Adaptation behavior was different across sessions (Fig. 5c; nonparetic propulsion: $p=0.032, \mathrm{t}(11)=2.46, \mathrm{~d}=0.98$, paretic propulsion: $p=0.015, \mathrm{t}(10)=-2.94, \mathrm{~d}=1.23)$, the changes in propulsion forces from early to late Adaptation were similar across sloped conditions (Fig. 5d; nonparetic propulsion: $p=0.92, \mathrm{t} \quad(11)=0.10, \mathrm{~d}=0.02$, paretic propulsion: $p=0.33, \mathrm{t}(10)=-1.04, \mathrm{~d}=0.33$ ). While paretic propulsion After-Effects are similar in either sloped conditions (Fig. 5e, $p=0.43, \mathrm{t}(10)=.82, \mathrm{~d}=$ $0.17)$, the non-paretic After-Effects are larger in magnitude following incline adaptation $(p=0.015, \mathrm{t}(11)=2.90$, $\mathrm{d}=0.82$ ). Note that the paretic propulsion forces change the most during Adaptation (Fig. 5d), whereas the nonparetic propulsion forces are the ones exhibiting aftereffects during Post-Adaptation (Fig. 5e). In summary, incline walking results in augmented paretic propulsion forces during Adaptation and reduced non-paretic propulsion force After-Effects.

\section{Discussion}

\section{Summary}

We investigated the effect of locomotor propulsion demands on motor adaptation and recalibration of gait in

A
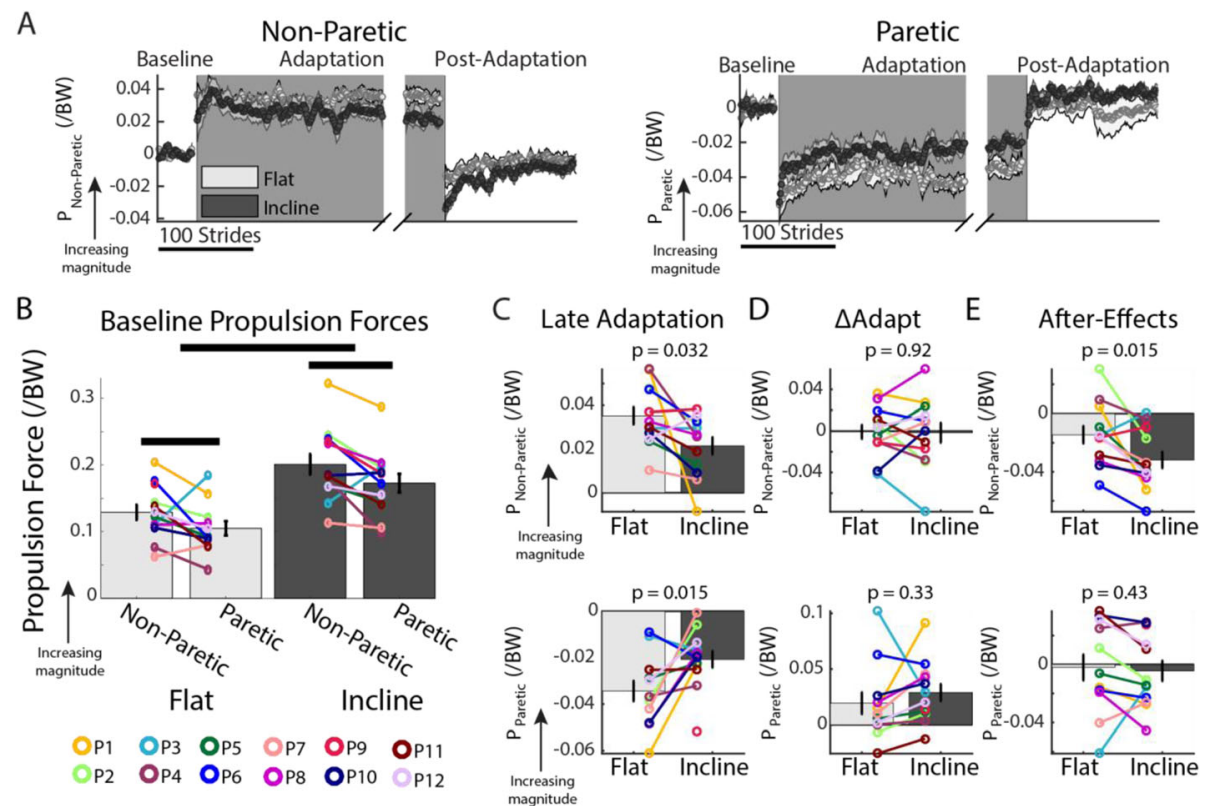

Fig. 5 Propulsion force Adaptation and After-Effects. a Stride-by-stride time courses of propulsion forces of the non-paretic (top panel) and paretic leg (bottom panel) are shown during self-selected Baseline, Adaptation, and Post-Adaptation. Note that each subject's baseline bias has been removed, resulting in average propulsion values of zero during Baseline. Each data point represents the average of 5 consecutive strides and shaded regions indicate the standard error for each group. For display purposes only, we include stride values during Post-Adaptation that were computed with a minimum of 10 subjects and the late adaptation behavior is aligned to the end of each subject's adaptation epoch. $\mathbf{b}$-e We display group average values for propulsion force outcome measures \pm standard errors. Individual subjects are represented with colored dots connected with lines. b Baseline: Thick horizontal black lines indicated that there is a significant effect of leg (i.e., paretic or non-paretic) and slope (i.e., flat or incline) on propulsion forces. On average, stroke subjects generate larger propulsion forces with their non-paretic leg, and they generate larger propulsion forces with both legs when walking incline. However, some individual stroke subjects generate larger propulsion forces with their paretic than their non-paretic leg. c Late Adaptation: Stroke subjects were closer to their baseline propulsion forces in the incline than the flat sessions. Moreover, baseline propulsion forces in the incline session were larger than the flat session (Fig. 2c). Taken together, these results suggest that stroke subjects are forced to propel more during incline split-belt walking with both legs compared to flat split-belt walking. d $\Delta$ Adapt: Propulsion forces were similarly modulated during the Adaptation epoch for both sloped conditions. e After-Effects: Even though both sloped sessions did not change the extent of propulsion force adaptation ( $\Delta$ Adapt), slope influenced the After-Effects for the non-paretic leg, but not the paretic leg 
the asymmetric motor system by altering the slope of the split-belt walking surface (i.e., flat vs. incline conditions). Survivors of a stroke adapted their step length asymmetry more in the incline than the flat condition resulting in Late Adaptation step length asymmetries that were smaller in magnitude (i.e., more successfully recovered baseline step length asymmetry). We also found that the speed-specific leg orientations (i.e., $\alpha$ and $X$ ) for both legs during Adaptation were predictive of those Post-Adaptation, leading to greater step length asymmetry after-effects in the incline than flat sessions. Lastly, larger step length asymmetry after-effects resulted from shorter paretic step lengths and lower non-paretic propulsion forces during Post-Adaptation in the incline session. In summary, the ability to control leg orientation to meet speed and force demands during split-belt walking is maintained post-stroke, which can be exploited for designing effective gait rehabilitation interventions.

\section{Post-stroke gait adapts more in response to larger propulsion demands}

We found that survivors of a stroke behaved similarly to young, intact adults in their response to sloped split-belt walking [70]. Specifically, survivors of a stroke were able to augment their propulsion forces in response to incline split-belt walking as observed in young, healthy adults [70]. It should be noted that all our analysis was done with peak forces, but we found similar effects with other metrics to quantify propulsion, such as impulse or mean force (data not shown). Our observation is consistent with previous literature indicating that patients in the chronic phase post-stroke can modulate paretic propulsion forces in response to task demands [3, 24-26, 34]. In particular, we observed during split-belt walking (i.e., adaptation period) that individuals exhibited larger nonparetic and paretic propulsion forces relative to baseline and early adaptation, respectively. These increments are possibly enabled by augmenting latent central drive to plantarflexors [2]. Interestingly, we find significant reduction in the fast (i.e. non-paretic) leg's propulsion forces post-adaptation (i.e., after-effects) relative to baseline walking. This finding is consistent with our previous study in young, unimpaired adults [70], but not with other studies reporting no significant changes in propulsion forces following split-belt walking in the flat condition $[57,66]$. We speculate that this might be because we use more naturalistic walking speeds than in previous experimental designs $[57,66]$.

Our results provide further evidence that the adaptation of step length asymmetry can be predicted from Baseline walking. Notably, it has been previously suggested that patients' gait asymmetries during baseline walking determine the extent to which they can adapt their movements in the unusual split condition [50]. We observed that survivors of a stroke reach distinct asymmetry levels across the incline and flat conditions. Thus, our results support previous findings $[40,41]$ indicating that it is not baseline gait asymmetry, but kinetic demands that govern the degree to which patients adapt their motor patterns in the split-belt task. More specifically, survivors of a stroke adjusted their leg orientations to augment the propulsion forces required for walking in the incline split condition as observed in young, healthy adults [70]. In other words, the forces generated to propel one's body forward constitute an important control variable regulating the adaptation of movements in the intact and asymmetric motor systems. Further, a recent study suggests that individuals adjust leg orientations to harness energy from the treadmill in the split condition [67]. While this theory explains well the orientation of the leading leg, it does not match well the observed orientation of the trailing leg (Additional file). Perhaps, other factors, such as stability [10], also contribute to the control of leg orientation in walking. It should be pointed out that we only tested the relation between leg orientations during baseline and adaptation in the paretic leg. We speculate that the same would have been observed in both legs and sloped conditions like young adults [70]. This is a reasonable expectation given that survivors of a stroke exhibited similar control of leg orientations to young adults during Late Adaptation and early Post-Adaptation for both legs and sloped conditions. Nonetheless, future work is needed to verify that the relation between baseline and adaptation is observed for distinct inclination conditions, or if the legs were walking at different belt-speed ratios than the ones we used.

\section{Bilateral adaptation in survivors of a stroke contrasts unilateral adaptation in young adults}

Survivors of a stroke recruited both legs in order to adapt their gait, whereas young adults primarily adapted one leg. Notably, we observed that survivors of a stroke adapted both the paretic (slow belt) and non-paretic (fast belt) step lengths, whereas we previously found that young individuals predominantly adjusted the fast belt step length $[60,70]$. This could be because survivors of a stroke may require more repetitions in the altered environment [75] to recover their baseline leg orientation with their paretic leg, whereas intact subjects can do so immediately after the split condition is introduced. Alternatively, it could be that the larger neural coupling post-stroke [35] enhances bilateral adaptation. In other words, it might not be possible for individuals who had experienced a stroke to adapt one leg in isolation due to neural drive sent to both limbs. Regarding postadaptation, paretic after-effects were only observed in 
the incline condition. More specifically, paretic step lengths become longer than in baseline walking, which may be beneficial for survivors of a stroke who take short paretic step lengths [4]. On the other hand, nonparetic after-effects were observed regardless of the sloped condition. This was atypical since the non-paretic leg walked fast in the split condition and young adults only exhibit after-effects in the leg that walked slow [70]. Thus, it was unexpected to observe non-paretic step lengths shorter than those taken during baseline. This shortening of non-paretic step lengths might be a strategy to recover balance (e.g., [18]), which is challenged upon removal of the split condition [10, 29]. In summary, survivors of a stroke adapt both legs during splitbelt walking, but paretic step length after-effects are only observed following incline split-belt walking.

\section{Neurorehabilitation through reinforcement of a corrective pattern during adaptation, rather than short-lived after- effects post-adaptation}

The long-term therapeutic effect of locomotor adaptation with split-belt treadmills may be due to walking with the motor demands of the split-belt task, rather than the adaptation effects observed post-adaptation. Split-belt walking has been shown to reduce long term gait asymmetry [7, 47, 61]. Little is known about the aspects of split-belt walking that underlie these long-term changes: the cumulative effect of brief after-effects postadaptation or the repeated exposure to motor demands specific to the split condition during the adaptation period. After-Effects could lead to motor improvements [5] such as temporarily reduced gait asymmetry [12, 62]. However, these after-effects are short lived and decrease as individuals experience multiple days of practicing the split-belt condition $[38,45,71]$. It is known that regular treadmill walking cannot modify gait asymmetries poststroke $[33,58,69]$, suggesting that the specific motor demands of the split-belt task might be important for neurorehabilitation. For example, we observe that the split condition (during the adaptation period) forces patients to take longer paretic step lengths and generate greater paretic propulsion forces. Perhaps practice of these gait features through multiple exposures to the split situation reinforces those patterns and ultimately leads to long-term reductions of gait symmetry overground. It is also possible that the strenuous nature of split-belt walking increases neural plasticity, as shown with other high-intensity exercises [1]. Thus, incline split-belt walking may be beneficial not only for inducing greater paretic propulsion, but also because it is more demanding than level walking [30]. Lastly, the initial disruption of step length asymmetry might trigger exploration of new locomotor patterns [52] that could converge to more metabolically efficient gait than their baseline walking pattern $[67,68]$. In summary, the longterm benefit of split-belt walking may originate from practicing and reinforcing motor patterns imposed by the split condition, rather than repeating the briefly lived after-effects.

\section{Clinical implications}

Split-belt walking has been shown to induce long term changes that could improve the mobility of those who have had a stroke $[7,47,61]$. Previous studies have investigated the impact of training design (e.g., paretic leg on the slow belt vs. fast belt) on the therapeutic effect of split-belt walking $[40,61]$. Our work contributes to this literature by indicating that this choice depends on the rehabilitation outcome of interest. For instance, our results suggest that placing the paretic leg on the fast belt would force subjects to augment their paretic propulsion forces and lengthen their paretic steps during split-belt walking, which could be advantageous to some patients. This idea is consistent with a pilot study showing that platarflexor moments trained on the fast side increase following multiple split-belt walking sessions [8]. This therapeutic effect is beneficial to individuals with baseline asymmetries due to shorter paretic step lengths coupled with plantar flexor weakness [6]. It remains, however, an open question the extent to which incline split-belt walking could augment paretic propulsion when the paretic leg is placed on the fast belt. Future studies are needed to test this given the limited changes in paretic propulsion that we observed post-adaptation compared to controls [70]. In sum, our study provides greater understanding of the motor demands associated to the split-belt task, which could be harnessed for gait neurorehabilitation.

Incline split-belt training may be a promising way to augment locomotor and adaptation and recalibration in the lesioned motor system. Not everyone who has had a stroke re-learns to walk symmetrically following several weeks of flat split-belt training [7, 47, 61]. Thus, it is clinically relevant to explore alternative strategies to augment adaptation in survivors of stroke other than increasing the speed difference [7, 77] since not all patients can walk with large speed differences. While this work indicates that adaptation can be augmented in patients, previous work indicates that overground walking post-stroke is most improved following decline, rather than incline, interventions [11]. Moreover, it has been previously observed that motor patterns observed on the treadmill do not fully transfer to overground walking $[63,71,73,74]$. Thus, future studies are needed to determine if the augmented adaptation in the incline environment transfers to flat overground walking. Furthermore, we designed our study to evaluate changes in step length asymmetry relative to baseline walking. It is, 
however, clinically relevant to determine if the augmented adaptation and after-effects lead to reduced step length asymmetry in absolute terms. Finally, it must be noted that participants were moderately to mildly impaired (i.e, $21<$ Fugl-Meyer Assessment leg motor score $\leq 34)$. Therefore, it remains an open question if similar results could be observed in more severely affected individuals or with different demographics than our cohort of participants.

\section{Conclusion}

We investigated the influence of augmenting propulsion demands during walking on the plasticity of locomotion post-stroke. We found that individuals who have suffered a stroke adapt their gait more during split-belt walking and have greater after-effects post-adaptation when propulsion demands are increased by inclining the treadmill. Like intact subjects, after-effects are predicted by each participant's leg orientations achieved during split-belt walking, which in turn is predicted by subjectspecific leg orientations during baseline walking. These results have two implications. First, these findings indicate that survivors of a stroke can adjust their movements to meet kinetic demands imposed by the walking condition. Second, subject-specific baseline behavior can predict the extent to which people will adjust their movements during and after the split condition. Taken together, our findings contribute to existing literature investigating mechanisms underlying locomotor adaptation induced by split-belt walking, which could be exploited for designing effective gait rehabilitation interventions post-stroke.

\section{Supplementary information}

Supplementary information accompanies this paper at https://doi.org/10 1186/s12984-020-00698-y.

Additional file 1: Figure S1. Adaptation and After-Effects of the leading leg and training leg positions. (A) Stride-by-stride time courses of leg positions ( $a$ and $X$ ) for the non-paretic and paretic leg are shown during self-selected Baseline, Adaptation, and Post-Adaptation. Each data point represents the average of 5 consecutive strides and shaded regions indicate the standard error for each group. The beginning and Late Adaptation group average behavior are shown for the Adaptation epoch. For display purposes only, we include stride values during Post-Adaptation that were computed with a minimum of 10 subjects. (B) Schematic of the self-selected Baseline, early Adaptation, and late Adaptation behavior for the paretic and non-paretic leg orientations, respectively. Note that during the adaptation period, the leading positions (a) increase for both legs, whereas the training position $(X)$ increases for the paretic leg (slow leg) and drecreases for the non-paretic leg (fast leg).

\section{Abbreviations}

SL: step length; a: leading leg position relative to the hip at leading leg heel strike; $X$ : trailing leg position relative to the hip at leading leg heel strike; GRF: ground reaction forces; AP: anterior-posterior; P Pareitc: paretic peak propulsion forces; $\mathrm{P}_{\text {Non-Pareitc: }}$ non-paretic peak propulsion forces; MRI: magnetic resonance imaging

\section{Acknowledgements}

The authors acknowledge the valuable input from Pablo Iturralde and Digna de Kam.

\section{Authors' contributions}

G.T.-O. and C.S. were involved with the conception and design of the work. C.S. collected and analyzed the data. C.S. and G.T.-O. interpreted the results. C.S. drafted the manuscript, which was carefully revised by all authors. The final version of the manuscript has been approved by all the authors.

\section{Funding}

C.S. is funded by a fellowship from the National Science Foundation (NSFGRFP); CRDF 4.30204; NSF1535036; AHA 15SDG25710041. APC charges for this article were fully paid by the University Library System, University of Pittsburgh.

\section{Availability of data and materials}

The datasets used and/or analyzed during the current study are available at https://osf.io/vm597/.

\section{Ethics approval and consent to participate}

Written and informed consent was obtained from all participants prior to participation. The University of Pittsburgh Institutional Review Board approved the used experimental protocol.

\section{Consent for publication}

No applicable.

\section{Competing interests}

The authors declare that they have no competing interests.

Received: 9 September 2019 Accepted: 21 May 2020

Published online: 03 June 2020

\section{References}

1. Andrews SC, Curtin D, Hawi Z, Wongtrakun J, Stout JC, Coxon JP. Intensity matters: high-intensity interval exercise enhances motor cortex plasticity more than moderate exercise. Cereb Cortex. 2019;30(1):1-12.

2. Awad LN, Hsiao HY, Binder-Macleod SA. Central drive to the paretic ankle Plantarflexors affects the relationship between propulsion and walking speed after stroke. J Neurol Phys Ther. 2020;44(1):42-8.

3. Awad LN, Reisman DS, Kesar TM, Binder-Macleod SA. Targeting paretic propulsion to improve poststroke walking function: a preliminary study. Arch Phys Med Rehabil. 2014;95(5):840-8.

4. Balasubramanian CK, Bowden MG, Neptune RR, Kautz SA. Relationship between step length asymmetry and walking performance in subjects with chronic hemiparesis. Arch Phys Med Rehabil. 2007:88(1):43-9.

5. Bastian AAJ. Understanding sensorimotor adaptation and learning for rehabilitation. Curr Opin Neurol. 2008;21(6):628-33.

6. Betschart M, Lauzière S, Miéville C, McFadyen BJ, Nadeau S. Changes in lower limb muscle activity after walking on a split-belt treadmill in individuals post-stroke. J Electromyogr Kinesiol. 2017;32(2017):93-100.

7. Betschart M, McFadyen BJ, Nadeau S. Repeated split-belt treadmill walking improved gait ability in individuals with chronic stroke: a pilot study. Physiother Theory Pract. 2018a;34(2):81-90.

8. Betschart M, McFayden BJ, Nadeau S. Lower limb joint moments on the fast belt contribute to a reduction of step length asymmetry over ground after split-belt treadmill training in stroke: A pilot study. Physiother Theory Pract 2018b;00(00):1-11.

9. Bowden MG, Balasubramanian CK, Neptune RR, Kautz S. a. Anterior-posterior ground reaction forces as a measure of paretic leg contribution in Hemiparetic walking. Stroke. 2006;37(3):872-6.

10. Buurke TJW, Lamoth CJC, Vervoort D, van der Woude LHV, den Otter R. Adaptive control of dynamic balance in human gait on a split-belt treadmill. J Exp Biol. 2018;221(13):jeb174896.

11. Carda S, Invernizzi M, Baricich A, Cognolato G, Cisari C. Does altering inclination alter effectiveness of treadmill training for gait impairment after stroke? A randomized controlled trial. Clin Rehabil. 2013;27(10):932-8.

12. Choi JT, Vining EPG, Reisman DS, Bastian AJ. Walking flexibility after hemispherectomy: split-belt treadmill adaptation and feedback control. Brain. 2009;132(Pt 3):722-33 [cited 2014 Jul 21]. 
13. Daly JJ, Zimbelman J, Roenigk KL, McCabe JP, Rogers JM, Butler K, et al. Recovery of coordinated gait: randomized controlled stroke trial of functional electrical stimulation (FES) versus no FES, with weight-supported treadmill and over-ground training. Neurorehabil Neural Repair. 2011;25(7): 588-96

14. Day KA, Leech KA, Roemmich RT, Bastian AJ. Accelerating locomotor savings in learning: compressing four training days to one. J Neurophysiol. 2018;119(6):2100-13.

15. Dewolf AH, Ivanenko Y, Zelik KE, Lacquaniti F, Willems PA. Kinematic patterns while walking on a slope at different speeds. J Appl Physiol. 2018; 125(2):642-53

16. Dewolf AH, Ivanenko YP, Lacquaniti F, Willems PA. Pendular energy transduction within the step during human walking on slopes at different speeds. PLoS One. 2017;12(10):1-25.

17. Dunlap WP, Cortina JM, Vaslow JB, Burke MJ. Meta-analysis of experiments with matched groups or repeated measures designs. Psychol Methods. 1996;1(2):170-7.

18. Eng JJ, Winter DA, Patla AE. Strategies for recovery from a trip in early and late swing during human walking. Exp Brain Res. 1994;102(2):339-49.

19. Finley JM, Bastian AJ, Gottschall JS. Learning to be economical: the energy cost of walking tracks motor adaptation. J Physiol. 2013;591(4):1081-95.

20. Finley JM, Long A, Bastian AJ, Torres-Oviedo G. Spatial and temporal control contribute to step length asymmetry during Split-Belt adaptation and Hemiparetic gait. Neurorehabil Neural Repair. 2015;29(8):786-95.

21. Finley JM, Statton M. A, Bastian AJA. A novel optic flow pattern speeds splitbelt locomotor adaptation. J Neurophysiol. 2014;111(5):969-76.

22. Fox SM, Haskell WL. The exercise stress test: needs for standardization. Cardiol Curr Top Prog. Eliakim M. New York: Academic Press; 1970. p. 149-154.

23. Fox SM, Naughton JP, Haskell WL. Physical activity and the prevention of coronary heart disease. Ann Clin Res. 1971:3:404-32.

24. Hsiao HY, Higginson JS, Binder-Macleod SA. Baseline predictors of treatment gains in peak propulsive force in individuals poststroke. J Neuroeng Rehabil; 2016a;13(1):2.

25. Hsiao HY, Knarr BA, Higginson JS, Binder-macleod SA. Mechanisms to increase propulsive force for individuals poststroke. J Neuroeng Rehabil. 2015:12(40):40.

26. Hsiao HY, Zabielski TM, Palmer JA, Higginson JS, Binder-Macleod SA. Evaluation of measurements of propulsion used to reflect changes in walking speed in individuals poststroke. J Biomech. 2016b;49(16):4107-12.

27. Hussain SJ, Morton SM. Perturbation schedule does not alter retention of a locomotor adaptation across days. J Neurophysiol. 2014;111(12):2414-22.

28. Item-glatthorn JF, Casartelli NC, Maffiuletti NA. Reproducibility of gait parameters at different surface inclinations and speeds using an instrumented treadmill system. Gait Posture. 2016:44:259-64.

29. Iturralde PA, Torres-Oviedo G. Corrective Muscle Activity Reveals SubjectSpecific Sensorimotor Recalibration. Eneuro. 2019;6(2):ENEURO.0358-18.2019.

30. Johnson AT, Benhur Benjamin M, Silverman N. Oxygen consumption, heat production, and muscular efficiency during uphill and downhill walking. Appl Ergon. 2002;33(5):485-91.

31. Jørgensen HS, Nakayama H, Raaschou HO, Olsen TS. Recovery of walking function in stroke patients: the Copenhagen stroke study. Arch Phys Med Rehabil. 1995 Jan;76(1):27-32.

32. Jørgensen L, Crabtree NJ, Reeve J, Jacobsen BK. Ambulatory level and asymmetrical weight bearing after stroke affects bone loss in the upper and lower part of the femoral neck differently: bone adaptation after decreased mechanical loading. Bone. 2000;27(5):701-7.

33. Kautz SA, Duncan PW, Perera S, Neptune RR, Studenski SA. Coordination of hemiparetic locomotion after stroke rehabilitation. Neurorehabil Neural Repair. 2005;19(3):250-8.

34. Kesar TM, Reisman DS, Perumal R, Jancosko AM, Higginson JS, Rudolph KS, et al. Combined effects of fast treadmill walking and functional electrical stimulation on post-stroke gait. Gait Posture. 2011:33(2):309-13.

35. Kloter E, Wirz M, Dietz V. Locomotion in stroke subjects: interactions between unaffected and affected sides. Brain. 2011;134(3):721-31.

36. Kwakkel G, Veerbeek JM, van Wegen EEH, Wolf SL. Constraint-induced movement therapy after stroke, Lancet Neurol. 2015;14(2):224-34.

37. Lamont EV, Zehr EP. Task-specific modulation of cutaneous reflexes expressed at functionally relevant gait cycle phases during level and incline walking and stair climbing. Exp Brain Res. 2006;173(1):185-92.

38. Larish DD, Martin PE, Mungiole M. Characteristic patterns of gait in the healthy old. Ann N Y Acad Sci. 1988;515(1):18-32.
39. Lauzière S, Miéville C, Betschart M, Aissaoui R, Nadeau S. Plantarflexor weakness is a determinant of kinetic asymmetry during gait in poststroke individuals walking with high levels of effort. Clin Biomech. 2015; 30(9):946-52.

40. Lauzière S, Miéville C, Betschart M, Duclos C, Aissaoui R, Nadeau S. Plantarflexion moment is a contributor to step length after-effect following walking on a split-belt treadmill in individuals with stroke and healthy individuals. J Rehabil Med. 2014;46:849-57.

41. Lauzière S, Miéville C, Betschart M, Duclos C, Aissaoui R, Nadeau S. A more symmetrical gait after split-belt treadmill walking increases the effort in paretic plantar flexors in people post-stroke. J Rehabil Med. 2016;48(7):576-82.

42. Lay AN, Hass CJ, Gregor RJ. The effects of sloped surfaces on locomotion: a kinematic and kinetic analysis. J Biomech. 2006;39(9):1621-8.

43. Lay AN, Hass CJ, Nichols TR, Gregor RJ. The effects of sloped surfaces on locomotion: an electromyographic analysis. J Biomech. 2007;40(6):1276-85.

44. Leech KA, Roemmich RT, Bastian AJ. Creating flexible motor memories in human walking. Sci Rep. 2018a;8(1):1-10.

45. Leech KA, Roemmich RT, Day KA, Bastian AJ. Movement and perception recalibrate differently across multiple days of locomotor learning. J Neurophysiol. 2018b;114(1):608-23.

46. Leroux A, Fung J, Barbeau H. Postural adaptation to walking on inclined surfaces: I. Normal strategies Gait Posture. 2002;15:64-74.

47. Lewek MD, Braun $\mathrm{CH}$, Wutzke $\mathrm{C}$. The role of movement errors in modifying spatiotemporal gait asymmetry post stroke: a randomized controlled trial. Clin Rehabil. 2018;32(2):161-72

48. Long AW, Finley JM, Bastian AJ, Aw L, Jm F, A BAJ. A marching-walking hybrid induces step length adaptation and transfers to natural walking 2015:3905-3914.

49. Long AW, Roemmich RT, Bastian AJ. Blocking trial-by-trial error correction does not interfere with motor learning in human walking. J Neurophysiol. 2016:115(5):2341-8.

50. Malone LA, Bastian AJ. Spatial and temporal asymmetries in gait predict Split-Belt adaptation behavior in stroke. Neurorehabil Neural Repair. 2014; 28(3):230-40.

51. Malone LA, Bastian AJ. Age-related forgetting in locomotor adaptation. Neurobiol Learn Mem. 2016;128:1-6.

52. Marchal-Crespo L, Schneider J, Jaeger L, Riener R. Learning a locomotor task: With or without errors? J Neuroeng Rehabil. 2014;11(25):25.

53. Mawase F, Haizler T, Bar-Haim S, Karniel A. Kinetic adaptation during locomotion on a split-belt treadmill. J Neurophysiol. 2013;109(8):2216-27 [cited 2014 Apr 20].

54. Mclntosh AS, Beatty KT, Dwan LN, Vickers DR, Ã ASM, Beatty KT, et al. Gait dynamics on an inclined walkway J Biomech 2006:39:2491-2502.

55. Mitchell AJ. The mini-mental state examination (MMSE): an update on its diagnostic validity for cognitive disorders. In: Larner AJ, editor. Cogn screen instruments a Pract approach. London: Springer; 2013. p. 15-46.

56. Morton SM, Bastian AJ. Cerebellar contributions to locomotor adaptations during splitbelt treadmill walking. J Neurosci. 2006;26(36):9107-16 [cited 2014 Jul 21].

57. Ogawa T, Kawashima N, Ogata T, Nakazawa K. Predictive control of ankle stiffness at heel contact is a key element of locomotor adaptation during split-belt treadmill walking in humans. J Neurophysiol. 2014;111(4):722-32 [cited 2014 Apr 3].

58. Den Otter AR, Geurts ACH, Mulder TH, Duysens J. Gait recovery is not associated with changes in the temporal patterning of muscle activity during treadmill walking in patients with post-stroke hemiparesis. Clin Neurophysiol. 2006;117(1):4-15

59. Patterson KK, Parafianowicz I, Danells CJ, Closson V, Verrier MC, Staines WR, et al. Gait asymmetry in community-ambulating stroke survivors. Arch Phys Med Rehabil. 2008:89(2):304-10.

60. Reisman DS, Block HJ, Bastian AJ, Darcy S, Block HJ, Bastian AJ. Interlimb coordination during locomotion: what can be adapted and stored? J Neurophysiol. 2005;94(4):2403-15 [cited 2013 Jun 20].

61. Reisman DS, McLean H, Keller J, Danks KA, Bastian AJ. Repeated split-belt treadmill training improves poststroke step length asymmetry. Neurorehabil Neural Repair. 2013;27(5):460-8.

62. Reisman DS, Wityk R, Silver K, Bastian AJ. Locomotor adaptation on a splitbelt treadmill can improve walking symmetry post-stroke. Brain. 2007;130(7): 1861-72 [cited 2014 Jul 12]. 
63. Reisman DS, Wityk R, Silver K, Bastian AJ. Split-belt treadmill adaptation transfers to overground walking in persons poststroke. Neurorehabil Neural Repair. 2009;23(7):735-44.

64. Rikli RE, Jones CJ. The reliability and validity of a 6-minute walk test as a measure of physical endurance in older adults. J Aging Phys Act. 1998;6(4): 363-75.

65. Roelker SA, Bowden MG, Kautz SA, Neptune RR. Paretic propulsion as a measure of walking performance and functional motor recovery post-stroke: a review. Gait posture [internet]. Elsevier. 2019;68:6-14.

66. Roemmich RT, Stegemöller EL, Hass CJ. Lower extremity sagittal joint moment production during split-belt treadmill walking. J Biomech. 2012; 45(16):2817-21.

67. Sánchez N, Simha SN, Donelan JM, Finley JM. Taking advantage of externa mechanical work to reduce metabolic cost: the mechanics and energetics of split-belt treadmill walking. J Physiol. 2019;15.JP277725.

68. Selinger JC, Shawn M, Connor O, Wong JD, Maxwell J, Selinger JC, et al. Humans Can Continuously Optimize Energetic Cost during Walking Report Humans Can Continuously Optimize Energetic Cost during Walking. Curr Biol. 2015;25(18):2452-6.

69. Silver K, Macko R, Forrester L, Goldberg A, Smith G. Effects of aerobic treadmill training on gait velocity, cadence, and gait symmetry in chronic hemiparetic stroke: a preliminary report. Neurorehabil Neural Repair. 2000; 14(1):65-71

70. Sombric CJ, Calvert JS, Torres-Oviedo G. Large propulsion demands increase locomotor learning at the expense of step length symmetry. Front Physiol. 2019;10:60.

71. Sombric CJ, Harker HM, Sparto PJ, Torres-oviedo G. Explicit action switching interferes with the context-specificity of motor memories in older adults. Front Aging Neurosci. 2017;9:40.

72. Statton MA, Vazquez A, Morton SM, Vasudevan EVL, Bastian AJ. Making sense of cerebellar contributions to perceptual and motor adaptation. Cerebellum The Cerebellum. 2018;17(2):111-21.

73. Torres-Oviedo G, Bastian AJ. Seeing is believing : effects of visual contextual cues on learning and transfer of Locomotor adaptation. J Neurosci. 2010; 30(50):17015-22.

74. Torres-Oviedo G, Bastian AJ. Natural error patterns enable transfer of motor learning to novel contexts. J Neurophysiol. 2012;107(1):346-56 [cited 2014 Feb 7].

75. Tyrell CM, Helm E, Reisman DS. Learning the spatial features of a locomotor task is slowed after stroke. J Neurophysiol. 2014;112(2):480-9 [cited 2014 Dec 18].

76. Wall-Scheffler CM, Chumanov E, Steudel-Numbers K, Heiderscheit B. Electromyography activity across gait and incline: the impact of muscular activity on human morphology. Am J Phys Anthropol. 2010;143(4):601-11.

77. Yokoyama H, Sato K, Ogawa T, Yamamoto S-I, Nakazawa K, Kawashima N. Characteristics of the gait adaptation process due to split-belt treadmill walking under a wide range of right-left speed ratios in humans. PLoS One. 2018;13(4):e0194875

\section{Publisher's Note}

Springer Nature remains neutral with regard to jurisdictional claims in published maps and institutional affiliations.

Ready to submit your research? Choose BMC and benefit from:

- fast, convenient online submission

- thorough peer review by experienced researchers in your field

- rapid publication on acceptance

- support for research data, including large and complex data types

- gold Open Access which fosters wider collaboration and increased citations

- maximum visibility for your research: over $100 \mathrm{M}$ website views per year

At $\mathrm{BMC}$, research is always in progress.

Learn more biomedcentral.com/submissions 